\title{
Indicadores cronológico, morfológico e funcional e os estágios da maturidade em escolares do nordeste do Brasil: um estudo comparativo
}

Vera Lúcia Bruch ${ }^{1}$, André Boscatto ${ }^{1}$, João Batista da Silva ${ }^{1}$, Asdrúbal Nóbrega, Montenegro Neto ${ }^{1}$, Humberto Jefferson de Medeiros $^{1,2}$, Paulo Moreira da Silva Dantas ${ }^{1,3}$ e Maria Irany Knackfuss ${ }^{1,4}$

1 - Laboratório de Biociências da Motricidade Humana-UFRN-RN

2 - Universidade do Estado do Rio Grande do Norte-RN

3 - UNIGRANRIO-RJ

4 - Universidade Federal do Rio Grande do Norte-RN

Bruch, V.; André Boscatto, A.; Silva, J. B.; Nóbrega, A.; Neto, M.; Medeiros, H. J.; Dantas, P. M.; Knackfuss, M. I.; Indicadores cronológico, morfológico e funcional e os estágios da maturidade em escolares do nordeste do Brasil: um estudo comparativo. Motricidade 3(1): 315-322

\section{Resumo}

O objetivo deste estudo centra-se na analise dos indicadores cronológico, morfológico e funcional de escolares da cidade de Mossoró-RN, a partir dos estágios maturacionais. $\mathrm{O}$ estudo caracterizou-se como uma pesquisa descritiva, com tipologia comparativa, a amostra limitou-se a $\mathrm{N}=305$ divididos em $\mathrm{n}=$ meninos 153 e $\mathrm{n}=$ meninas 152, de nove a 17 anos. Os protocolos utilizados foram IMC, somatória de dobras, salto horizontal e o teste de $30 \mathrm{~m}$. A estatística utilizada foi à descritiva. Os resultados apontam para o desenvolvimento que estará sempre atrelado ao crescimento. A existência do comportamento de modificação das variáveis estudadas, dentro de um caminho cronológico, mas com o corte maturacional, constitui-se em uma estratégia interessante de observação, permitindo, levar-se em conta a intervenção natural oriunda do crescimento e desenvolvimento dos indivíduos. Recomenda-se portanto que sejam levados em conta não somente o comportamento cronológico das variáveis intervenientes no crescimento e desenvolvimento humano, mas o comportamento maturacional, como também a utilização de instrumentos de indicação hereditária e genética.

Palavras-chave: maturação, qualidades fisicas básicas, crescimento e desenvolvimento.

Data de submissão: Outubro 2006

Data de aceitação: Dezembro 2006

\section{Abstract}

Chronological, morphological and functional markers and maturational stages of students from the northeast of Brazil: a comparative study.

The aim of this study was to analyse chronological, morphologic and functional markers of students in the city of Mossoró-RN, in Brazil, taking into account the maturational stages. The study was classified as a descriptive comparative research. The sample was composed of 305 children divided in two groups: $\mathrm{n}=$ masculine 153 and $\mathrm{n}=$ feminine 152 , from nine to 17 years. The protocols used were Body Mass Index, Skinfolds addiction, horizontal jump and the $30 \mathrm{~m}$ test. We used a descriptive statistical analysis. The results pointed to a development that will always be linked to the growth. The existence of behavior of modification of the studied variables, into a chronological way, but with the maturational cut, consists in an interesting strategy of observation, and it permit to take in account the natural intervention of the growth and development of the individuals. So, it is recommended that not only the chronological behavior of the intervening variables in the growth and human development be taken in account, but the maturacional behavior, as well as, the use of instruments of hereditary and genetic indication.

Key-words: maturation, basic physical qualities, growth and development. 


\section{Introdução}

A escola em nosso país possui divisões bastante caracterizadas. O ensino público, seja municipal, estadual ou federal, é distinto do privado e estas diferenças ficam claras em diversos setores: econômico, social, estrutural e organizacional. Tais diferenças influenciam sob maneira não só na qualidade do ensino, como no resultado oriundo desta escola ${ }^{1}$.

Em consonância com tais afirmações, está a realidade das populações que se servem destas instituições, caracterizadas por diferentes camadas sociais, e que não permitem aos estudos a generalização das observações.

Medeiros ${ }^{1}$ aponta que os indicadores ligados a saúde, vem despertando interesse de pesquisadores nas diferentes áreas de atuação, e os escolares tornam-se a população mais visada para desenvolver estes estudos.

Este tipo de estudo se faz necessário, principalmente na comunidade escolar, onde os instrumentos utilizados, os testes de qualidade fisica e a composição corporal, são alguns dos indicadores para avaliar o estado de saúde e o desenvolvimento físico destes escolares, clientela onde frequentemente tem-se observado indivíduos com sobrepeso ou obesidade.

Estes dois fatores são preocupantes porque estão associados a doenças como hipertensão, diabetes, doenças coronarianas, acidente vascular cerebral, osteoporose e altos níveis de colesterol sanguiíneo 2,3 .

Pena, Bacallao ${ }^{4}$ salientam que a redução na prática de exercícios físicos, decorrente da falta de oportunidade de praticá-los de modo regular e da ausência de informações no tocante aos benefícios associados e a modificação qualitativa na dieta das populações urbanas, com aumento no consumo de gorduras e redução no consumo de fibras, o que contribui para o aumento da prevalência de obesidade na população de baixa renda.
Observando as tendências epidemiológicas, fica evidente a necessidade de intervenção nesse relevante agravo à saúde, nos planos, coletivo e individual, abordando-se os principais fatores que modulam seu aparecimento, especialmente a atividade fisica e os hábitos alimentares ${ }^{5}$.

O contexto aqui exposto constitui-se como indicativo a realização do estudo proposto, indo ao encontro da necessidade da investigação quanto as variáveis relacionadas tanto a composição corporal como as capacidades funcionais do jovem escolar, constituindo-se portando o objetivo deste estudo, a analise dos indicadores cronológico, morfológico e funcional de escolares da cidade de MossoróRN, a partir dos estágios maturacionais estabelecidos por Medeiros ${ }^{1}$ para escolares da região do estado do Rio Grande do Norte, que utilizou estágios maturacionais de Tanner.

\section{Metodologia}

\section{Amostra}

$\mathrm{O}$ estudo se caracterizou como uma pesquisa descritiva, com tipologia comparativa, sendo os sujeitos distribuídos em grupos de acordo com as tabelas normativas do estágio da maturidade proposto por Medeiros ${ }^{1}$, para escolares do Rio grande do norte, estabelecendo como critério de analise, os pelos pubianos.

A amostra, de caráter não probabilístico intencional, limitou-se a 305 sujeitos (Meninos $=153 \mathrm{e}$ meninas $=152$ ), matriculados nas escolas, da rede estadual, localizadas na zona urbana do município de Mossoró, do estado do Rio Grande do Norte, Brasil. Foram incluídos neste estudo, escolares de $5^{a}$ a $8^{a}$ serie, numa faixa etária de 9 a 17 anos, participantes das aulas de Educação Física, que de forma voluntária, se disponibilizaram em participar dos procedimentos avaliativos; autorizados por seus responsáveis e que não apresentavam 


\section{Resultados}

algum tipo de enfermidade não foram inseridos neste estudo.

Os procedimentos usados neste estudo respeitam as normas internacionais de experimentação com humanos (Declaração de Helsínque, 1975) e do Comitê de Ética do Conselho nacional de Saúde do Brasil 196/96 . (colocar estes parágrafo na amostra).

\section{Procedimentos}

Os protocolos utilizados foram de Quetelet para determinação do IMC e Lohman para a somatória de dobras tricipital e subscapular referida em Fernandes Filho ${ }^{6}$.

$\mathrm{Na}$ avaliação do desempenho motor foi utilizado o salto horizontal para medir a força dos membros inferiores ${ }^{7}$, e o teste de $30 \mathrm{~m}$ para mensurar a velocidade ${ }^{8}$.

Para os cortes maturacionais por estágios utilizou-se o estudo de Medeiros ${ }^{1}$, que utilizou o Auto Tanner, validado por Matsudo ${ }^{9}$, dividindo os grupos em (1) pré-púbere, (2) púberes e (3) pós-púberes, esta divisão em três classes visou diminuir o erro interno.

A estatística utilizada foi à descritiva observando os valores de tendência central e seus derivados, associada a um teste de normalidade de Komogorov-Smirnov e Shapiro-Wilk. Para as comparações utilizou-se a Anova one-way e como posthoc o teste de scheffe para grupos de quantitativos diferentes. Objetivando-se a medição dos testes, o presente trabalho se pauta em consonância às considerações básicas do tratamento estatístico, a fim de manter-se a cientificidade da pesquisa, em que se considere o nível de significância de $\mathrm{p}<0,05$, isto é, 95\% de probabilidade para as afirmativas e/ou negativas, denotadas durante as investigações.
As Tabelas 1 e 2 demonstram-se os resultados de idade, estatura e massa, do masculino e feminino respectivamente.

Nas Tabelas 3 e 4, ao observar-se conjuntamente o IMC e a Soma das dobras de tríceps e subscapular, testes de Salto horizontal e corrida de 30 metros no masculino e feminino.

\section{Discussão}

As Tabelas 1 e 2 demonstram o comportamento esperado de aumento da idade tanto no masculino quanto para o feminino, independente do descritor de tendência central. Chama-se a atenção que houve sempre tendência a idades superiores no masculino, indicando maior aceleração das meninas quanto ao processo maturacional, o que corrobora com a literatura ${ }^{9,1,10}$ confirmando que a divisão nos três estágios obedeceu, Medei$\operatorname{ros}^{1}$, e que os resultados médios de entrada, saída e permanência nos estágios púberes, coadunamse com o referido autor.

A estatura e massa no masculino, obedece ao esperado quanto aceleração mais acentuado da saída da puberdade para pós-pubere, o que pode ser um indicado de maior manifestação das características secundárias masculinas oriundas de um grande aporte hormonal masculino ${ }^{11}$, e o feminino indica um comportamento proporcionalmente menor mais acentuado pelo aumento do peso.

Nas Tabelas 3 e 4, ao observar-se conjuntamente o IMC e a Soma das dobras de tríceps e subscapular no masculino e feminino, fica evidente nos grupos observados que a maior intervenção das características secundárias masculinas e femininas manifestam-se especialmente no aumenta da gordura dos compartimentos demonstrada nas espessura das dobras no feminino, já que, o IMC 
Indicadores cronológico, morfológico e funcional e os estágios da maturidade em escolares do nordeste do Brasil: um estudo comparativo

Vera Lúcia Bruch, André Boscatto, João Batista da Silva, Asdrúbal Nóbrega, Montenegro Neto, Humberto Jefferson de Medeiros, Paulo Moreira da Silva Dantas e Maria Irany Knackfuss

\begin{tabular}{lllll}
\hline \multicolumn{2}{l}{ Tabela 1: Masculino } & & & \\
\hline estagio & & idade & estatura & massa \\
\hline 1.00 & Mean & 9.9545 & 1.4086 & 38.8636 \\
& $\mathrm{~N}$ & 22 & 22 & 22 \\
& Std. Devitation & 0.21320 & 0.05401 & 9.77507 \\
& Median & $* 10.0000$ & 1.4000 & 34.5000 \\
\hline \multirow{2}{*}{3.00} & Mean & 11.9655 & 1.4902 & 41.7931 \\
& N & 87 & 87 & 87 \\
& Std. Devitation & 0.70626 & 0.103967 & 11.18987 \\
& Median & $* 12.0000$ & 1.4700 & $* 39.0000$ \\
& Mean & 14.9545 & 1.6251 & 54.0682 \\
& N & 44 & 44 & 44 \\
& Sta. Devitation & 1.01052 & 0.09587 & 12.18157 \\
& Median & $* 15.0000$ & 1.6350 & 52.5000 \\
& Mean & 12.5359 & 1.5173 & 44.9020 \\
& N & 153 & 153 & 153 \\
& Std. Devitation & 1.84970 & 0.12089 & 12.69468 \\
& Median & 12.0000 & 1.5000 & 44.0000 \\
\hline
\end{tabular}

*As variáveis dentro das classes após o teste de normalidades foram consideradas não paramétricas. Existência de diferenças para $p<0,01$ : Idade entre todos os estágios; estatura entre todos os estágios e massa $(1 / 3$ e $2 / 3)$.

\begin{tabular}{|c|c|c|c|c|}
\hline estagio & & idade & estatura & massa \\
\hline \multirow[t]{4}{*}{1.00} & Mean & 10.0000 & 1.3972 & 39.1111 \\
\hline & $N$ & 18 & 18 & 18 \\
\hline & Std. Devitation & 0.0000 & 0.06182 & 9.42393 \\
\hline & Median & * 100000 & 1.3950 & 38.5000 \\
\hline \multirow[t]{4}{*}{2.00} & Mean & 12.4286 & 1.4972 & 42.8295 \\
\hline & $\mathrm{N}$ & 105 & 105 & 105 \\
\hline & Std. Devitation & 1.47320 & 0.07878 & 9.56755 \\
\hline & Median & $* 12.0000$ & *1.4800 & $* 41.000$ \\
\hline \multirow[t]{4}{*}{3.00} & Mean & 13.3793 & 1.5483 & 49.4138 \\
\hline & $\mathrm{N}$ & 29 & 29 & 29 \\
\hline & Sta. Devitation & 0.49380 & 0.05305 & 9.46994 \\
\hline & Median & $* 13.000$ & 1.5500 & $* 46.000$ \\
\hline \multirow[t]{4}{*}{ Total } & Mean & 12.3224 & 1.4951 & 443.6454 \\
\hline & $\mathrm{N}$ & 152 & 152 & 152 \\
\hline & Std. Devitation & 1.55091 & 0.08316 & 9.94878 \\
\hline & Median & 12.0000 & 1.4900 & 42.0000 \\
\hline
\end{tabular}

*As variáveis dentro das classes após o teste de normalidades foram consideradas não paramétricas. Existência de diferenças para $\mathrm{p} \leftarrow 0,01$ : Idade entre todos os estágios; estatura entre todos os estágios e massa $(1 / 3$ e $2 / 3)$. 


\begin{tabular}{|c|c|c|c|c|c|}
\hline $\begin{array}{l}\text { Tabela } 3 \\
\text { estagio }\end{array}$ & & IMC & somaTRSE & saltoH & Velocidade \\
\hline \multirow[t]{4}{*}{1.00} & Mean & 19.4273 & 20.4545 & 1.4536 & 6.1614 \\
\hline & $\mathrm{N}$ & 22 & 22 & 22 & 22 \\
\hline & $\begin{array}{l}\text { Std. } \\
\text { Devitation }\end{array}$ & 4.17763 & 8.30219 & 0.23579 & 0.61803 \\
\hline & Median & * 17.600 & 20.0000 & 1.4000 & 6.1200 \\
\hline \multirow[t]{4}{*}{2.00} & Mean & 18.5276 & 16.7011 & 1.5544 & 5.9495 \\
\hline & $\mathrm{N}$ & 87 & 87 & 87 & 87 \\
\hline & $\begin{array}{l}\text { Std. } \\
\text { Devitation }\end{array}$ & 3.37269 & 6.96188 & 0.22401 & 0.66105 \\
\hline & Median & *17.900 & $* 16.000$ & 1.5600 & $* 6.140$ \\
\hline \multirow[t]{4}{*}{3.00} & Mean & & 14.9545 & 1.6251 & 54.0682 \\
\hline & $N$ & 44 & 44 & 44 & 44 \\
\hline & $\begin{array}{l}\text { Sta. } \\
\text { Devitation }\end{array}$ & 3.22283 & 6.01302 & 0.25180 & 1.35424 \\
\hline & Median & *19.850 & $* 16.0000$ & 1.7050 & $* 5.4600$ \\
\hline \multirow[t]{4}{*}{ Total } & Mean & 19.850 & 12.5359 & 1.5173 & 44.9020 \\
\hline & $\mathrm{N}$ & 153 & 153 & 153 & 153 \\
\hline & $\begin{array}{l}\text { Std. } \\
\text { Devitation }\end{array}$ & 3.52042 & 6.98685 & 0.25220 & 0.90776 \\
\hline & Median & 18.3000 & 16.0000 & 1.5900 & 6.0600 \\
\hline
\end{tabular}

*As variáveis dentro das classes após o teste de normalidades foram consideradas não paramétricas.

Existência de diferenças para $p \leftarrow 0,01$ : IMC (2/3); Salto Horizontal(1/3 e 2/3).

guarda muito mais a relação de volume em quilogramas por metro quadrado, do que indicativo evidenciando um aumento ponderal significativo. Tais afirmativas corroboram com a literatura quanto ao aumento ponderal e discordam com a mesma quanto à utilização do IMC de maneira isolada como indicativo de maior obesidade ${ }^{12}$. Fica assim marcada para o grupo em foco que existe no feminino uma tendência acentuada do aumento ponderal e estabilização do mesmo para o masculino.

Ainda nas Tabelas 3 e 4, quanto a força e velocidade, avaliadas respectivamente pelos testes de Salto horizontal e corridade30 metros. Observase maior evolução da qualidade força no mascu- lino, o que pode ser a confirmação da manifestação hormonal masculina já assinalada em outras características secundárias do grupo, reforçada por um aumento menos marcado do feminino ${ }^{13}$ já a velocidade não acentua esta diferença o que pode ser um indicativo de maior intervenção coordenativa, já que, o deslocamento em velocidade requer maiores níveis de atributos Motores ${ }^{14}$.

Quanto à existência ou não de diferenças significativas, nas meninas somente nas características cronológicas e morfológicas observa-se tais diferenças estatísticas, o que poderia ser mais um indicativo de aceleração maturacional antes assinalada ${ }^{15}$, em tempo é bom que se diga que a não existência de uma diferença significativa não é 
Indicadores cronológico, morfológico e funcional e os estágios da maturidade em escolares do nordeste do Brasil: um estudo comparativo

Vera Lúcia Bruch, André Boscatto, João Batista da Silva, Asdrúbal Nóbrega, Montenegro Neto, Humberto Jefferson de Medeiros, Paulo Moreira da Silva Dantas e Maria Irany Knackfuss

\begin{tabular}{|c|c|c|c|c|c|}
\hline estagio & & IMC & somaTRSE & saltoH & Velocidade \\
\hline \multirow[t]{4}{*}{1.00} & Mean & 19.8889 & 28.6667 & 1.2983 & 7.0856 \\
\hline & $\mathrm{N}$ & 18 & 18 & 18 & 18 \\
\hline & $\begin{array}{l}\text { Std. } \\
\text { Devitation }\end{array}$ & 3.75184 & 15.63923 & 0.15120 & 0.75641 \\
\hline & Median & 19.6500 & $* 24.000$ & 1.2750 & 7.1050 \\
\hline \multirow[t]{4}{*}{2.00} & Mean & 18.5276 & 16.7011 & 1.5544 & 5.9495 \\
\hline & $\mathrm{N}$ & 105 & 105 & 105 & 104 \\
\hline & $\begin{array}{l}\text { Std. } \\
\text { Devitation }\end{array}$ & 3.10161 & 11.17406 & 0.22706 & 0.85899 \\
\hline & Median & $* 18.800$ & $* 22.000$ & $* 1.3100$ & *6.5700 \\
\hline \multirow[t]{4}{*}{3.00} & Mean & 20.5931 & 22.5172 & 1.4490 & 6.5190 \\
\hline & $\mathrm{N}$ & 29 & 29 & 29 & 29 \\
\hline & $\begin{array}{l}\text { Sta. } \\
\text { Devitation }\end{array}$ & 3.93173 & 11.47239 & 0.25981 & 0.70988 \\
\hline & Median & *19.300 & *19.000 & 1.4700 & 6.5200 \\
\hline \multirow[t]{4}{*}{ Total } & Mean & 19.3605 & 24.8750 & 1.3628 & 6.7723 \\
\hline & $\mathrm{N}$ & 152 & 152 & 152 & 151 \\
\hline & $\begin{array}{l}\text { Std. } \\
\text { Devitation }\end{array}$ & 3.39575 & 11.86252 & 0.22942 & 0.83063 \\
\hline & Median & 19.0000 & 21.0000 & 1.3350 & 7.0000 \\
\hline
\end{tabular}

*As variáveis dentro das classes após o teste de normalidades foram consideradas não paramétricas.

indicativa de estagnação de comportamento de uma variável. No masculino tanto nas variáveis cronológicas quanto morfológicas e funcionais foi observada a existência de diferenças, o que em linhas gerais e corroborando com a afirmativa anterior, demonstra maior cinesia quanto ao efeito hormonal do crescimento masculino.

Os resultados parecem apontar que o desenvolvimento estará sempre atrelado ao crescimento, pois não existem compartimentos estanques, ao se referenciar o ser humano. Por essa razão, a individualidade biológica é o marco destas observações e quanto mais instrumentos poderem ser utilizados neste caminho menos serão as chances de equívocos.

O estudo ora apresentado responde aos questionamentos inseridos em seus objetivos, ou seja, observa-se um comportamento de aceleração no masculino e frenagem no feminino, quanto as variáveis indicativas de performance na execução de tarefas motoras, ditadas por maior força, estatura, e conseqüente maior aceleração feminina e frenagem masculina, quanto a características de aumento da massa gorda, diferenças estas em grande parte induzidas pelos hormônios sexuais. A existência do comportamento de modificação das variáveis estudadas, dentro de um caminho cronológico, mas com o corte maturacional, constitui-se em uma estratégia interessante de observação, permitindo, levar-se em conta a intervenção natural oriunda do crescimento e desenvolvimento dos indivíduos.

Recomenda-se portanto que sejam levados em conta não somente o comportamento cronoló- 


\section{Referências}

gico das variáveis intervenientes no crescimento e desenvolvimento humano, mas o comportamento maturacional, como também a utilização de instrumentos de indicação hereditária e genética.

\section{Correspondência}

\section{Vera Lúcia Bruch}

Rua Marechal Serejo 601 b.07/202

Jacarepaguá/RJ - Brasil.

CEP: 22743-380.

vbruch@terra.com.br
1. Medeiros HJ, Santos DB, Rego SAJS, Mila ASB; Farias AS; Knackfuss MI; Fernandes Filho J. (2005) Características dermatoglíficas dos escolares nos diferentes estágios maturacionais no Estado do Rio Grande do Norte. . In: Novena Jornada de Educacion Física del Mercosur 2005. 55:89-93.

2. Benetti M; Rebelo FPV; Carvalho T. (2000) Regressão da aterosclerose coronariana. Rev Bras Ativ Fís Saúde. 5(3):58-75.

3. Blake GJ; Ridker PM. (2002) Inflammatory bio-markers and cardiovascular risk prediction. $J$ Intern Med. 252(4):283-294.

4. Peña M, Bacallao J. (2000) La obesidade en la pobreza: um problema emergente em lãs americas. In: la obesidade en la pobreza: un nuevo reto para la salud pública Organización Panamericana de la Salud -OPS. 576:3-12.

5.Vasconcelos VL, Gisela AP. (2003) Overweight and obsity prevalences in male adolescentes in northcast Brazil, 1980 - 2000. Cad Saúde Pública. 19(5):1445-51.

6. Fernandes Filho J. (2003) A prática da avaliação física: Testes, medidas e avaliação física em escolares, atletas e academias de ginástica. ( $\left.2^{\mathrm{a}} \mathrm{ed}\right)$. Rio de Janeiro: Carpe.

7. Matsudo VKR. (1987) Testes em Ciências do Esporte. ( $4^{\circ}$ ed). São Caetano do Sul: Centro de Estudos do Laboratório de Aptidão Física de São Caetano do Sul.

8. Matsudo VCR. (1982) Testes em ciências do esporte. São Paulo: Centro de Estudos do Laboratório de Aptidão Física de São Caetano do Sul.

9. Matsudo SMM; Matsudo VKR. (1991) Validade da auto-avaliação na determinação da maturação sexual. Rev Bras Ciênc Mov. 5(2):18-35.

10. Alonso LV; SILVA Dantas PM. et al. (2003) Perfil somatotípico e dermatoglífico de tipologia de fibra muscular de atletas mirins da equipe de futsal do clube de regatas Vasco da Gama - RJ. 
Indicadores cronológico, morfológico e funcional e os estágios da maturidade em escolares do nordeste do Brasil: um estudo comparativo

Vera Lúcia Bruch, André Boscatto, João Batista da Silva, Asdrúbal Nóbrega, Montenegro Neto, Humberto Jefferson de Medeiros, Paulo Moreira da Silva Dantas e Maria Irany Knackfuss

Rev Bras Ciênc Mov. 11(4):121-123.

11. Tourinho Filho H; Tourinho LSPR. (1998) Crianças, adolescentes e atividade física: aspectos maturacionais e funcionais. Rev paul Educ Fís. 12(1):71-84.

12. Giugliano R; Melo ALP. (2004) Diagnosis of overweight and obesity in schoolchildren: utilization of the body mass index international standard. J. Pediatr. 80(2):129-34.

13. Thomas JR, Nelson JK. (2002) Métodos de pesquisa em atividade física. Porto Alegre: Artmed.

14. Klein CMO; Fernandes Filho J. (2003) Relação entre a dermatoglifia, as qualidades físicas e o nível maturacional de escolares adolescentes de ambos os sexos. Fit \& Perform J. 2(6):321-329.

15. Tsukamoto MHC; Nunomura, M. (2003) Aspectos maturacionais em atletas de ginástica olímpica do sexo feminino. Motriz. 9(2):119-26. 